\title{
SOME THEOREMS CONCERNING PARTITIONS $\left({ }^{1}\right)$
}

\author{
BY \\ EMIL GROSSWALD
}

1. Introduction. Let $q$ be an odd prime and, unless another modulus is specifically indicated, let the congruence symbol denote congruences modulo $q$. Let $\{a\}=\left\{a_{1}, a_{2}, \cdots, a_{m}\right\}$ be a set of $m$ distinct least positive residues $\bmod q$. Such a set $\{a\}$ will be called symmetrical, if with $a$ also $q-a$ belongs to $\{a\}$. Furthermore, $\nu \equiv\{a\}$ shall mean that $\nu$ runs only through values congruent $(\bmod q)$ to some $a_{j}(j=1,2, \cdots, m)$. Define the function $F(x)$ $=\prod_{\nu \equiv\{a\}}\left(1-x^{n}\right)^{-1}=\sum_{n=0}^{\infty} p_{n}(q) x^{n}$. Then $p_{n}(q)$ represents the number of partitions of the integer $n$ into summands congruent to elements of $\{a\}$. Similarly, let $H(x)=\prod_{\nu \equiv\{a\}}\left(1+x^{\nu}+x^{2 v}+\cdots+x^{l v}\right)=\sum_{n=0}^{\infty} p_{n}(q, l) x^{n}$; then $p_{n}(q, l)$ represents the number of partitions of the integer $n$ into summands congruent to elements of $\{a\}$, no summand being repeated more than $l$ times. In case $q=5$ and $\{a\}$ is either the set of quadratic residues, or that of quadratic nonresidues, Lehner, using the method of Rademacher $[10 ; 11]$, obtained convergent series for $p_{n}(5)$ (see [5]). The first term of these series, being the dominant one, is asymptotically equal to $p_{n}(5)$. Lehner's result has been generalized by Livingood [6] for any prime $q>3$ and $\{a\}=(a, q-a), a \neq 0$. Recently, Petersson (see especially [8] and [9]), using an entirely different approach, obtained some very interesting results concerning these, and more general, partition numbers. In particular, he has obtained (see [8]) an expression for $p_{n}(q)$ and also a very precise asymptotic formula. By further exploiting his method he obtained [8] among others, the following result: Let the prime $q>5$ satisfy $q \equiv 1(\bmod 4)$ and denote by $p_{n}^{+}(q), p_{n}^{-}(q)$ the number of partitions of $n$ into quadratic residues and into quadratic nonresidues $(\bmod q)$, respectively. Let $h$ be the class number of the real quadratic field $R\left(q^{1 / 2}\right)$ and denote by $\epsilon>1$ its fundamental unit; denote also by $\alpha_{5}(q)$ the number of representations of $q$ by five squares, representations differing by sign, or order of summands being considered as distinct. Then, as $n \rightarrow \infty$,

$$
\frac{p_{n}^{+}(q)}{p_{n}^{-}(q)}=\epsilon^{h}\left(1-\frac{\pi}{6}\left(\frac{3(q-1)}{q}\right)^{1 / 2} c \cdot \alpha_{5}(q) n^{-1 / 2}+O\left(n^{-1}\right)\right)
$$

holds with $c=1 / 240$ if $q \equiv 1(\bmod 8)$ and $c=1 / 560$ if $q \equiv 5(\bmod 8)$. Similarly, if $p_{n}^{+}(q, l), p_{n}^{-}(q, l)$ are defined as above, with the additional restriction that

Presented to the Society, February 23, 1957; received by the editors February 19, 1957.

(1) The author is happy to acknowledge his indebtness to Professor N. Fine for many stimulating discussions, especially in the early stages of this investigation. 
no summand may occur more than $l$ times, then, for $n \rightarrow \infty$,

$$
\frac{p_{n}^{+}(q, l)}{p_{n}^{-}(q, l)}=1+\frac{\pi}{6}\left(\frac{3(q-1) l}{q(l+1)}\right)^{1 / 2} \cdot l c \alpha_{5}(q) n^{-1 / 2}+O\left(n^{-1}\right),
$$

$c$ being defined as in (1). (For comparison it should be observed that, while in general Petersson's notation has been adhered to, here $l$ is used for his $l-1$.)

2. In both the Rademacher and the Petersson method use is made of the modular character of the generating functions. Indeed, if $\{a\}$ is symmetrical and if we set $x=\exp (2 \pi i \tau / q)$, then $F(x)$ and $H(x)$ become modular functions (or forms) of $\tau$; hence, they remain (essentially) invariant if $\tau$ is replaced by $T(\tau)$, provided that $T$ is a transformation belonging to some subgroup $\bar{\Gamma}$ of finite index of the modular group $\Gamma$. Whenever applicable, both methods lead to exact representations for the partition numbers, which are the coefficients of $F$ and $H$. In case $\{a\}$ is not symmetric, $F$ and $H$ (as functions of $\tau$ ) are no more modular and above methods cannot be applied directly. One may observe, however (see [5] and [6]; also [8]) what happens already in the case of modular generating functions when $\tau \rightarrow T(\tau)$, with $T \in \Gamma$, but $T \in \bar{\Gamma}$. Although the generating function does not stay invariant, it is only transformed into another function, whose behavior for $|x| \rightarrow 1$, resembles closely that of the original function and can be controlled. One may consider the case of asymmetric sets $\{a\}$ as a limiting case, with $\bar{\Gamma}$ reduced to the identity. The results are not quite as satisfactory as in the case of modular functions and, instead of exact expressions, one obtains only asymptotic fomulae $\left({ }^{2}\right)$. Some results for asymmetric $\{a\}$ were already found $\left({ }^{2}\right)$ by G. Meinardus [7]. His method presents a certain similarity to that used in $\S \S 7-9$ of this paper (both are essentially saddle point methods), but makes no use of the transformation formulae. He thus obtains the leading term of our (17), in the particular case $m=1$, but without the restriction of $q$ to be a prime. He also obtains the leading term of Petersson's formula (1) and of our formula (19).

In what follows, we proceed in two steps: (a) Using the Hardy-Rademacher approach, the transformation formulae are established for $F(x)$ and $H(x)(\$ \S 4,5,6)$ by the method of residues; (b) having determined the behavior of the generating functions, their coefficients are obtained $(\S \S 8,9)$, using a lemma (established in \$7) essentially due to Hayman [3]. Finally, some particular cases are considered $(\$ 10)$ and the corollaries are proven $(\S \S 11,12)$.

3. The results may be summarized in the following theorems.

TheOREM 1. As $x \rightarrow 1$,

(2) See, however $[8$, p. 27] for Petersson's objection to this use of the term "asymptotic."

( 3 ) The author acknowledges his indebtedness to the referee and to Professor P. T. Bateman, for calling his attention to this important reference. 


$$
\begin{aligned}
F(x) & =\prod_{\nu \equiv\{a\}}\left(1-x^{\nu}\right)^{-1} \\
& =\prod_{j=1}^{m} E\left(\frac{n_{j}}{q}\right)(-q \log x)^{d} \exp \left\{\frac{\Lambda}{-\log x}+K(-\log x)+O\left(\log ^{2} x\right)\right\}, \\
H(x) & =\prod_{\nu \equiv\{a\}_{i \nu} \neq \prod_{(\bmod l+1)}}\left(1-x^{\nu}\right)^{-1} \\
& =(l+1)^{-d} \exp \left\{\frac{\mu}{-\log x}+K l(-\log x)+O\left(\log ^{2} x\right)\right\},
\end{aligned}
$$

with

$$
\begin{gathered}
d=\frac{1}{q} \sum_{j=1}^{m}\left(a_{j}-\frac{q}{2}\right), \quad K=\frac{1}{24 q} \sum_{j=1}^{m} A_{j}, \quad A_{j}=q^{2}-6 a_{j} q+6 a_{j}^{2}, \\
\Lambda=\frac{m \pi^{2}}{6 q}, \quad \mu=\frac{l \Lambda}{l+1},
\end{gathered}
$$

$\Gamma(x)$ the gamma-function and $E(x)=\Gamma(x)(2 \pi)^{-1 / 2}$.

Theorem 2. As $n \rightarrow \infty$

$$
\begin{aligned}
p_{n}(q) & =\frac{\omega}{q}\left(\frac{q \Lambda^{1 / 2}}{\lambda_{n}}\right)^{d+1} \exp \left(-\frac{d(d+2)}{4 \Lambda^{1 / 2} \lambda_{n}}\right) I_{1}\left(2 \Lambda^{1 / 2} \lambda_{n}\right)\left(1+O\left(n^{-1}\right)\right), \\
p_{n}(q, l) & =(l+1)^{d}\left(\mu^{1 / 2} / \nu_{n}\right) I_{1}\left(2 \mu^{1 / 2} \nu_{n}\right)\left(1+O\left(n^{-1}\right)\right),
\end{aligned}
$$

with $\lambda_{n}=(n-K)^{1 / 2}, \nu_{n}=(n+K l)^{1 / 2}, I_{1}(x)$ the Bessel function and

$$
\omega=\prod_{j=1}^{m} E\left(a_{j} / q\right)
$$

Corollary 1 (Petersson). If $q \equiv 1(\bmod 4)$ and $n \rightarrow \infty$, then (1) and (2) hold.

Corollary 2. If $q \equiv 3(\bmod 4)$ and $n \rightarrow \infty$, then

$$
\begin{aligned}
\frac{p_{n}^{+}(q)}{p_{n}^{-}(q)}= & \prod_{a \bmod q}\left\{\Gamma\left(\frac{a}{q}\right)\right\}^{(a / q)}\left(\frac{12 n}{\pi^{2} q(q-1)}\right)^{h / w} \\
& \cdot\left(1+\frac{1}{\pi}\left(\frac{6 q}{q-1}\right)^{1 / 2} \frac{h}{w} n^{-1 / 2}+O\left(n^{-1}\right)\right), \\
\frac{p_{n}^{+}(q, l)}{p_{n}^{-}(q, l)}= & (l+1)^{h}\left(1+O\left(n^{-1}\right)\right) .
\end{aligned}
$$

Here the exponent $(a / q)$ stands for the Legendre symbol, while $h$ is the class number of the field $R\left((-q)^{1 / 2}\right)$ and $w$ the number of its roots of unity. 
Finally, we may remark that Corollary 1 shows that (1) holds for $q=5$ (not covered by Petersson's theorem); however this (and much more) follows already from Lehner's result [5], simply by taking into account two or more terms of the asymptotic expansion of the Bessel function $I_{1}(t)$.

4. Proof of Theorem 1. In order to determine the behaviour of $F(x)$ and $H(x)$ near $x=1$ we shall use a simplified version of the method of Hardy and Rademacher. The method itself has already been presented several times in great detail (see $[2 ; 10 ; 11 ; 5])$; therefore, it will be sufficient only to outline the procedure.

For $a \neq 0$ define $F_{a}(x)=\prod_{\nu \equiv a}\left(1-x^{\nu}\right)^{-1}$. Then

$$
G_{a}(x)=\log F_{a}(x)=-\sum_{\nu \equiv a} \log \left(1-x^{\nu}\right)=\sum_{n=1}^{\infty} \sum_{m=0}^{\infty} n^{-1} x^{(q m+a) n} .
$$

Setting $x=e^{-2 \pi z}, R z>0$, and applying Mellin's theorem, one obtains

$$
\begin{aligned}
G_{a}(x) & =\sum_{n=1}^{\infty} \sum_{m=0}^{\infty} n^{-1} \exp \{-2 \pi z(q m+a) n\} \\
& =\sum_{n=1}^{\infty} \sum_{m=0}^{\infty}(2 \pi i)^{-1} \int_{(\alpha)} \Gamma(s) d s /(2 \pi z)^{s}(q m+a)^{s} n^{s+1} \\
& =\frac{1}{2 \pi i} \int_{(\alpha)} \frac{\Gamma(s) \zeta(s, a / q) \zeta(s+1)}{(2 \pi)^{s} z^{s} q^{s}} d s .
\end{aligned}
$$

Here $\zeta(s, a / q)$ and $\zeta(s+1)$ are Hurwitz's and Riemann's zeta functions, respectively, and the integrals are taken along a parallel to the imaginary axis, of abscissa say $\alpha=3 / 2$. Using the functional equation of the Hurwitz zeta function,

$$
\begin{aligned}
\zeta(s, a / q)=2 \Gamma(1-s)(2 \pi q)^{s-1}\left\{\sin \frac{\pi s}{2} \sum_{\nu=1}^{q} \cos \frac{2 \pi a \nu}{q} \zeta\left(1-s, \frac{\nu}{q}\right)\right. \\
\left.+\cos \frac{\pi s}{2} \sum_{\nu=1}^{q} \sin \frac{2 \pi a \nu}{q} \zeta\left(1-s, \frac{\nu}{q}\right)\right\}
\end{aligned}
$$

one obtains

$$
\begin{array}{r}
G_{a}(x)=\frac{1}{4 \pi i q} \int_{(\alpha)}\left\{\sum_{\nu=1}^{q} \frac{\cos (2 \pi a \nu / q)}{\cos (\pi s / 2)} z^{-s \zeta}\left(1-s, \frac{\nu}{q}\right) \zeta(s+1)\right. \\
\left.\quad+\sum_{\nu=1}^{q} \frac{\sin (2 \pi a \nu / q)}{\sin (\pi s / 2)} z^{-s \zeta}\left(1-s, \frac{\nu}{q}\right) \zeta(s+1)\right\} d s .
\end{array}
$$

Changing $s$ into $-s$, 


$$
\begin{aligned}
G_{a}(x)=\frac{1}{4 \pi i q} \int_{(-\alpha)}\left\{\sum_{\nu=1}^{q} \frac{\cos (2 \pi a \nu / q)}{\cos (\pi s / 2)} z^{s} \zeta\left(1+s, \frac{\nu}{q}\right) \zeta(1-s)\right. \\
\left.\quad-\sum_{\nu=1}^{q} \frac{\sin (2 \pi a \nu / q)}{\sin (\pi s / 2)} z^{s} \zeta\left(1+s, \frac{\nu}{q}\right) \zeta(1-s)\right\} d s .
\end{aligned}
$$

We may shift the line of integration back to the abscissa $\alpha$, by taking into account the residues of the poles between the two lines of integration. It follows that $G_{a}(x)=(4 \pi i q)^{-1} \int_{(\alpha)}\left(I_{1}(s)+I_{2}(s)\right) d s-2 \pi i\left(R_{1}+R_{2}\right)$, where

$$
I_{1}(s)=\frac{1}{4 \pi i q} \sum_{\nu=1}^{q} \frac{\cos (2 \pi a \nu / q)}{\cos (\pi s / 2)} z^{s} \zeta\left(1+s, \frac{\nu}{q}\right) \zeta(1-s), I_{2}(s)
$$

is obtained from $-I_{1}(s)$, by replacing all cosines by sines, and $R_{i}(i=1,2)$ are the sums of the residues of the $I_{i}$ 's between the abscissae $-3 / 2$ and $3 / 2$. The integral can be estimated as follows: The functional equation of the Riemann zeta-function is applied to $\zeta(1-s)$, and (5) is used to replace

$$
\sum_{\nu=1}^{q}\left\{\cos \frac{2 \pi a \nu}{q} \zeta\left(1+s, \frac{\nu}{q}\right)-\sin \frac{2 \pi a \nu}{q} \zeta\left(1+s, \frac{\nu}{q}\right) \operatorname{cotg} \frac{\pi s}{2}\right\}
$$

by $-\zeta(-s, a / q)(2 \pi q)^{1+8} / 2 \sin (\pi s / 2) \Gamma(1+s)$; then the integral becomes

$$
\begin{aligned}
-\frac{1}{2 i} \int_{(\alpha)}(q z)^{s} \Gamma(s) \zeta(s) \zeta(-s, & \left.\frac{a}{q}\right) \frac{\operatorname{cosec} \pi s / 2}{\Gamma(1+s)} d s \\
& =-\frac{1}{2 i} \int_{(\alpha)} \frac{(q z)^{s}}{s \sin \pi s / 2} \zeta(s) \zeta\left(-s, \frac{a}{q}\right) d s=\psi(z) .
\end{aligned}
$$

As $\psi^{\prime}(z)=2^{-1} i \int_{(\alpha)}(q z)^{s-1} \operatorname{cosec}(\pi s / 2) \zeta(s) \zeta(-s, a / q) d s$ is absolutely convergent for $1<\alpha<2$, it follows that $\psi(z)$ is an analytic function of $z$, vanishing for $z=0$. Moving the line of integration beyond $\alpha=2$ and taking into account the residue at $s=2$, one obtains

$$
\psi(z)=\frac{q^{2} \pi^{2}}{6} \phi_{4}^{\prime}(a / q) z^{2}+\sum_{\nu=3}^{\infty} a_{v} z^{\nu} .
$$

From the analyticity of $\psi(z)$ follows the convergence of the second member for sufficiently small $z$. Here $\phi_{4}(t)$ stands for the fourth Bernoulli polynomial and all coefficients $a$, could be determined explicitly, but are not needed here. The computation of the residues uses mainly (5); most of the details can be found in [5] and [6]. $I_{1}(s)$ has simple poles at $s=-1$ and $s=1$ and an apparently double pole at $s=0$; let the corresponding residues be $R_{11}$, $R_{12}, R_{13}$. For $s=-1, \sum_{\nu=1}^{q} \cos (2 \pi a \nu / q) \zeta(0, \nu / q)=-1 / 2$, so that $R_{11}$ $=-1 / 24 i q z$. For $s=0$, 


$$
\begin{aligned}
\lim _{s \rightarrow 0} \sum_{\nu=1}^{q} \cos \frac{2 \pi a \nu}{q} & \zeta\left(1+s, \frac{\nu}{q}\right) \\
& =-2 q\left\{\left(\frac{1}{2}-\frac{a}{q}\right)(\log 2 \pi q+\gamma)-\log \Gamma\left(\frac{a}{q}\right)+\frac{1}{2} \log 2 \pi\right\}
\end{aligned}
$$

is regular; hence, $s=0$ is actually a simple pole. Dividing previous sum by $4 \pi i q$ one obtains $-R_{12}$. For $s=1$,

$$
\sum_{\nu=1}^{q} \cos \frac{2 \pi a \nu}{q} \zeta\left(2, \frac{\nu}{q}\right)=-2 \pi^{2} q^{2} \zeta\left(-1, \frac{a}{q}\right)=2 \pi^{2} q^{2}\left(a^{2} / 2 q^{2}-a / 2 q+1 / 12\right),
$$

so that the residue becomes $R_{13}=A z / 24 q i$ with $A=6 a^{2}-6 a q+q^{2} \cdot I_{2}(s)$ has only the (apparently triple) pole at $s=0$. However,

$$
\lim _{s \rightarrow 0} \sum_{\nu=1}^{q} \sin (2 \pi a \nu / q) \zeta(1+s, \nu / q)=\pi q(1 / 2-a / q)
$$

is regular, so that $s=0$ is actually a double pole. Its residue is found to be $R_{2}=(2 \pi i)^{-1}(1 / 2-a / q)(\log z-\gamma)$. Here, as in $R_{12}, \gamma$ stands for the Euler constant. Combining these results, $G_{a}(x)=-2 \pi i\left(R_{11}+R_{12}+R_{13}+R_{2}\right)+O\left(z^{2}\right)$ $=\pi / 12 q z-\pi A z / 12 q-\left\{(1 / 2-a / q) \log (2 \pi q z)-\log \Gamma(a / q)+2^{-1} \log 2 \pi\right\}+O\left(z^{2}\right)$. Returning now to $F_{a}(x)=\exp \left\{G_{a}(x)\right\}$ and to the variable $x$, we have

$F_{a}(x)=(2 \pi)^{-1} \Gamma(a / q)(-q \log x)^{(a / q-1 / 2)} \exp \left\{-\pi^{2}(6 q \log x)^{-1}\right.$

$$
\left.+A \log x /(24 q)+O\left(\log ^{2} x\right)\right\} \text {. }
$$

As $F(x)=\prod_{j=1}^{m} F_{a_{j}}(x)$, it follows that

(7) $F(x)=(2 \pi)^{-m / 2} \prod_{j=1}^{m} \Gamma\left(\frac{a_{j}}{q}\right)(-q \log x)^{d} \exp \left\{-\Lambda / \log x+K \log x+O\left(\log ^{2} x\right)\right\}$ with $\Lambda, d$ and $K$ as defined in $\S 3$. Observing that

$$
H_{a}(x)=\prod_{\nu \equiv a}\left(1+x^{\nu}+\cdots+x^{l \nu}\right)=F_{a}(x) / F_{a}\left(x^{l+1}\right),
$$

it follows from (7) and $H(x)=\prod_{j=1}^{m} H_{a_{j}}(x)$ that

$$
H(x)=(l+1)^{-d} \exp \left\{-\mu / \log x-K l \log x+O\left(\log ^{2} x\right)\right\},
$$

with $\mu=l \Lambda /(l+1)$. For $a \equiv 0, F_{0}(x)=\prod_{\nu \equiv 0}\left(1-x^{\nu}\right)^{-1}=\prod_{\nu=1}^{\infty}\left(1-x^{q \nu}\right)^{-1}$ and it is well known (it follows, e.g. from Lemma 4.31 in $[2$, p. 93]) that for $x \rightarrow 1$, $\prod_{n=1}^{\infty}\left(1-x^{n}\right)^{-1}=(-\log x / 2 \pi)^{1 / 2} \exp \left\{-\pi^{2}(6 \log x)^{-1}+(\log x) / 24+O\left(\log ^{2} x\right)\right\}$. 
Consequently, it follows that (7), hence also (8) holds even if one of the residues in $\{a\}$ satisfies $a \equiv 0$, provided one sets not $a=0$, but $a=q$, whence the condition of least positive residues. This finishes the proof of Theorem 1.

5. In what follows, it will be necessary to have some information concerning the behavior of $F(x)$ and $H(x)$ in the neighborhood of an arbitrary rational point on the circle of radius $|x|=r<1$, i.e. a point of argument $2 \pi h / k$. Let $x=r e^{i \theta}=\exp \{(-2 \pi z+2 \pi i h) / k\}, z=-k(\log r+2 \pi i \phi) / 2 \pi$ so that $\phi=\theta / 2 \pi$ $-h / k$. Proceding essentially as in $\$ 4$ (see also [6]) one finds that for $q \mid k$. $G_{a}(x)=\sum_{j=1}^{4}\left\{\int_{(\alpha)} I_{j}(s) d s-2 \pi i R_{j}\right\}$. Here

$$
\begin{aligned}
I_{1}(s)= & \frac{1}{4 \pi i k^{2}} \sum_{\rho, \mu, \nu=1}^{k} \cos \frac{2 \pi \rho \mu}{k} \cos \frac{2 \pi \rho h^{\prime} \nu}{k} \zeta\left(1-s, \frac{\mu}{k}\right) \zeta\left(1+s, \frac{\nu}{k}\right) z^{s} \sec \frac{\pi s}{2}, \\
I_{2}(s)= & -\frac{1}{4 \pi i k^{2}} \sum_{\rho, \mu, \nu=1}^{k} \cos \frac{2 \pi \rho \mu}{k} \sin \frac{2 \pi \rho h^{\prime} \nu}{k} \zeta\left(1-s, \frac{\mu}{k}\right) \zeta\left(1+s, \frac{\nu}{k}\right) z^{s} \\
& \cdot \operatorname{cosec} \frac{\pi s}{2}
\end{aligned}
$$

$I_{3}(s)$ is obtained from $i I_{2}(s)$, by changing each trigonometric function into its co-function and $I_{4}(s)$ is obtained in the same way from $i I_{1}(s)$; also, $R_{j}$ stands for the sum of the residues of $I_{j}(s)(j=1,2,3,4)$ inside the strip $(-3 / 2,3 / 2)$, while $h^{\prime}$ and $\rho$ are defined, respectively, by $h h^{\prime} \equiv-1(\bmod k)$ and $\rho \equiv-h a \equiv b(\bmod q), 0<h^{\prime}, \rho<k, 0<b<q$. One may remark that $I_{1}(s)$, $I_{4}(s)$, hence also $R_{1}, R_{4}$ are even functions of $a$, while $I_{2}, I_{3}, R_{2}$ and $R_{3}$ are odd functions. The integrals, estimated as in $\S 4$, are $O\left(z^{2}\right)$. For the residues one obtains

$$
R_{1}=(A z-B / z) / 24 i k q, R_{2}=i \phi_{a}(h, k) / \pi, R_{3}=C_{a}(h, k) z, R_{4}=-\sigma_{a}(h, k) / 2 .
$$

Here $A$ is defined as in $\S 3, B=q^{2}-6 q b+6 b^{2}$, while

$$
\begin{aligned}
& \sigma_{a}=\sum((\lambda / v k))((h \lambda / k)), \\
& C_{a}=-\frac{1}{12 \pi k^{2}} \sum \frac{\Gamma^{\prime}(u)}{\Gamma(u)}\left(6 \lambda^{2}-6 k v \lambda+k^{2} v^{2}\right), \\
& \phi_{a}=\sum((\lambda / v k))\left\{((\lambda h / k))(\log 2 \pi k+\gamma)-\log \Gamma(u)+\frac{1}{2} \log 2 \pi\right\}
\end{aligned}
$$

with $v=1, u=1 / 2-((\lambda h / k))$, the summations being extended over all values of $\lambda$ satisfying $\lambda \fallingdotseq a, 1 \leqq \lambda<v k, h \lambda \neq 0(\bmod k)$ and with $((y))$ defined as usual to be zero for integral $y,((y))=y-[y]-1 / 2$ otherwise. In case $q \nmid k$, the procedure has to be modified slightly, as in [5] and [6] and leads to $R_{1}$ $=\left(A z-z^{-1}\right) / 24 i k q$, while the other residues remain formally the same, provided that now one sets $v=q$ in (9), instead of $v=1$, as previously done. If we also define $B$ as before, when $q \mid k, B=1$ otherwise, all residues become for- 
mally the same and we may omit the case distinction $q \mid k$, vs. $q \nmid k$. Combining these results, $\log F_{a}(x)=-2 \pi i \sum_{j=1}^{4} R_{j}+O\left(z^{2}\right)$, whence

$$
\begin{aligned}
\log F_{a}(x)= & \frac{A}{24 q} \log r-\frac{\pi^{2} B}{6 q k^{2}} \frac{\log r}{\log ^{2} r+4 \pi^{2} \phi^{2}}+2 \phi_{a}-2 \pi C_{a} k \phi \\
& +i\left\{\left(\frac{A \pi}{12 q}+\frac{\pi^{3} B}{3 q k^{2}\left(\log ^{2} r+4 \pi^{2} \phi^{2}\right)}\right) \phi+C_{a} k \log r+\pi \sigma_{a}\right\} \\
& +O\left(\log ^{2} r+\phi \log r+\phi^{2}\right)
\end{aligned}
$$

follows. In particular, setting $L=\left(\pi^{2} / 6 q\right) \sum_{a \in\{a\}} B$ one obtains, as a consequence of (10),

$$
\begin{aligned}
|F(x)|= & \exp \left\{-\frac{L \log r}{k^{2}\left(\log ^{2} r+4 \pi^{2} \phi^{2}\right)}\right. \\
& \left.+K \log r+2 \Phi-2 \pi C k \phi+O\left(\log ^{2} r+\phi \log r+\phi^{2}\right)\right\}
\end{aligned}
$$

where $\Phi=\sum_{a \in\{a\}} \phi_{a}, C=\sum_{a \in\{a\}} C_{a}$. If $k=q s, s \geqq 1$, then, for $q>3, B=q^{2}$ $-6 b q+6 b^{2} \leqq q^{2} t, L \leqq \pi^{2} m q t / 6,0<L / k^{2} \leqq t \Lambda / s^{2} \leqq \Lambda t$ with $t=1-6(q-1) / q^{2}<1$. For $q=3, b=1,2, B=-3$ and $L<0$. If $q \nmid k$, then $B=1, L=\Lambda, L / k^{2}=\Lambda / k^{2}$ $\leqq \Lambda / 4$, for $k>1$. Therefore, if $k>1$, in all cases $L / k^{2} \leqq t \Lambda$; also, $K>-m q / 48$. Concerning $H(x)=F(x) / F\left(x^{l+1}\right)$ it follows from (10) that

$$
\begin{aligned}
|H(x)| \leqq & \exp \left\{\frac{-L \log r}{k^{2}\left(\log ^{2} r+4 \pi^{2} \phi^{2}\right)}-K l \log r+2\left(\Phi(h, k)-\Phi\left(h_{1}, k_{1}\right)\right)\right. \\
& -2 \pi\left(C(h, k) k \phi-C\left(h_{1}, k_{1}\right) k_{1} \phi_{1}\right) \\
& \left.+O\left(\log ^{2} r+\left(\phi+\phi_{1}\right) \log r+\phi^{2}+\phi_{1}^{2}\right)\right\}
\end{aligned}
$$

where $x_{1}=x^{l+1}$ and $h_{1}, k_{1}$ and $\phi_{1}$ are defined with respect to $x_{1}$, as $h, k$ and $\phi$ were defined with respect to $x$.

6. For further use we need the trivial estimates

$$
\Phi=O(k \log k), \quad C=O\left(k^{2}\right) .
$$

For that, set $((\lambda h / k))=y$. From the conditions on $\lambda$ and $h$ it follows that $|y| \leqq 1 / 2-1 / k$. One observes that within this range $U(y)=y(\log 2 \pi k+\gamma)$ $-\log \Gamma(1 / 2-y)+2^{-1} \log 2 \pi$ satisfies $-2^{-1} \log k-\gamma / 2+o(1)<U(y)<2^{-1} \log k$ $+\log \log k+(\log 2 \pi+\gamma / 2-1)+o(1)$. Hence, $\phi_{a}=\sum((\lambda / v k)) U(y)=O(k \log k)$. Similarly, for $1 / k \leqq u \leqq 1-1 / k$, from $\Gamma^{\prime}(u) / \Gamma(u)=-1 / u-\gamma+u \zeta(2)-u^{2} \zeta(3)$ $+\cdots$ follows that $-k-\gamma<\Gamma^{\prime}(u) / \Gamma(u)<-\gamma+\zeta(2)$. Also $\left|6 \lambda^{2}-6 k v \lambda+v^{2} k^{2}\right|$ $<v^{2} k^{2}$, so that $\left|\left(\Gamma^{\prime}(u) / \Gamma(u)\right)\left(6 \lambda^{2}-6 k v \lambda+v^{2} k^{2}\right)\right|<k^{3} v^{2}(1+o(1))$ and $C_{a}$ $=O\left(k^{-2} k^{4} v\right)=O\left(k^{2}\right)$ and (13) follows. One may observe that in the case of symmetric sets, (13) follows trivially from the previously remarked fact that $R_{2}$ and $R_{3}$ are odd functions of $a$, so that $C=\Phi=0$ for symmetric sets $\{a\}$.

7. In the proof of Theorem 2 we need the following 
Lemma. Let $f(x)=\sum_{n=0}^{\infty} a_{n} x^{n}$ be analytic inside the unit circle and define the functions $a(r)=r \cdot d(\log f(r)) / d r$ and $b(r)=r \cdot d a(r) / d r$ of $r=|x|$. Denote by $\rho=\rho_{n}$ the unique $\left.{ }^{4}\right)$ root of $a(\rho)=n$ and assume that for $r_{0}<r<1$, functions $\delta(r)>0$ and $u(r)$ exist, with the following properties: As $n \rightarrow \infty$, one has, for some $\alpha>0$ :

$$
\begin{aligned}
& \int_{|\theta| \geq \delta(\rho)}\left|f\left(\rho e^{i \theta}\right)\right| d \theta=O\left(n^{-\alpha} f(\rho) b(\rho)^{-1 / 2}\right) ; \\
& \text { (b) } \int_{-\delta(\rho)}^{+\delta(\rho)}\left(f\left(\rho e^{i \theta}\right)-f(\rho) \exp \left\{i \theta a(\rho)-\frac{1}{2} \theta^{2} b(\rho)\right\}\right) e^{-i n \theta} d \theta \\
& =(2 \pi)^{1 / 2} f(\rho) b(\rho)^{-1 / 2}\left(u(\rho)+O\left(n^{-\alpha}\right)\right) ; \\
& \delta(\rho)^{2} b(\rho) \geqq 2 \alpha \log n .
\end{aligned}
$$

Then

$$
a_{n}=\rho^{-n}(2 \pi b(\rho))^{-1 / 2} f(\rho)\left(1+u(\rho)+O\left(n^{-\alpha}\right)\right) .
$$

This lemma is essentially Hayman's Theorem 12 in [3]. The sharper estimates of the error terms were needed; hence, generality has been traded for precision.

Proof of the lemma. Integrating around the circle of radius $r<1$, by Cauchy's theorem,

$$
a_{n}=\frac{1}{2 \pi i} \int_{0}^{2 \pi} \frac{f\left(r e^{i \theta}\right)}{r^{n+1} e^{i(n+1) \theta}} r i e^{i \theta} d \theta
$$

consequently,

$$
\begin{aligned}
a_{n} r^{n}= & \frac{1}{2 \pi} \int_{0}^{2 \pi} f\left(r e^{i \theta}\right) e^{-i n \theta} d \theta=\frac{1}{2 \pi}\left[\int_{|\theta|>\delta} f\left(r e^{i \theta}\right) e^{-i n \theta} d \theta\right. \\
& +\int_{-\delta}^{+\delta}\left\{f\left(r e^{i \theta}\right)-f(r) \exp \left\{i \theta a(r)-\frac{1}{2} \theta^{2} b(r)\right\}\right\} e^{-i n \theta} d \theta \\
& \left.+f(r) \int_{-\delta}^{+\delta} \exp \left\{i \theta(a(r)-n)-\frac{1}{2} \theta^{2} b(r)\right\} d \theta\right] .
\end{aligned}
$$

For $r=\rho$ it follows from the definition of $\rho$ that the last integral becomes $\int_{-\delta}^{+\delta} e^{-\theta^{2} b / 2} d \theta=(2 / b(\rho))^{1 / 2} \int_{-D}^{+D} e^{-x^{2}} d x=(2 / b(\rho))^{1 / 2}\left(\pi^{1 / 2}-\epsilon\right)$ with $D=\delta(\rho)(b(\rho) / 2)^{1 / 2}$ and $\epsilon=\int_{D}^{\infty} e^{-x^{2}} d x<e^{-D^{2}} / 2 D$. The conclusion now follows by assumptions (a), (b) and (c) of the lemma and observing that, by (c), $0<\epsilon<n^{-\alpha}(8 \alpha \log n)^{-1 / 2}$ $=o\left(n^{-\alpha}\right)$.

8. Proof of Theorem 2. Observing that the term $O\left(\log ^{2} x\right)$ in (7) originates from (6), taking into account the convergence of the series in $\psi(z)$ and using the definitions of the lemma, one obtains for $F(x)$, that $a(r)=\Lambda / \log ^{2} r+d / \log r$

(4) For the uniqueness see $[3$, p. 67]. 
$+K+O(r \log r) ; b(r)=-2 \Lambda / \log ^{3} r-d / \log ^{2} r+O(1)$ and that $\rho=\rho_{n}$, the root of $a(\rho)=n$, satisfies:

$$
\begin{aligned}
(\log \rho)^{-1}= & -\left(\frac{n}{\Lambda}\right)^{1 / 2}\left(1+\frac{1}{2} d(\Lambda n)^{-1 / 2}+\frac{1}{2}\left(\frac{d^{2}}{4 \Lambda}-K\right) n^{-1}\right. \\
& \left.-\frac{1}{8}\left(\frac{d^{2}}{4 \Lambda}-K\right)^{2} n^{-2}+O\left(n^{-3}\right)\right), \\
\log \rho= & -\left(\frac{\Lambda}{n}\right)^{1 / 2}\left(1-\frac{1}{2} d(\Lambda n)^{-1 / 2}+\frac{1}{2}\left(\frac{d^{2}}{4 \Lambda}+K\right) n^{-1}\right. \\
& \left.+\frac{1}{2} d \Lambda^{-1 / 2}\left(\frac{d^{2}}{4 \Lambda}-K\right)^{2} n^{-3 / 2}+O\left(n^{-2}\right)\right) .
\end{aligned}
$$

Define $\delta=\delta(r)=(-\log r)^{3 / 2} \psi(r)$ with $\psi(r)=((-4 / \Lambda) \log (-\log r))^{1 / 2}$. Then $\delta(r)^{2} b(r)=-8 \log (-\log r)(1+O(\log r))$ and, for $r=\rho$, using $(15), \delta(\rho)^{2} b(\rho)$ $=4 \log n(1+o(1))>2 \log n$; hence, assumption (c) of the lemma holds with $\alpha=1$. Next, let $D(\rho, \theta)=\left(F\left(\rho e^{i \theta}\right)-F(\rho) \exp \left\{i \theta a(\rho)-2^{-1} \theta^{2} b(\rho)\right\}\right) e^{-i n \theta}$. Using (7), previous values of $a(r), b(r)$ and (15), expanding the exponentials, simplifying, taking into account that $|\theta| \leqq \delta(\rho)$ and regrouping terms, one obtains $D(\rho, \theta)=F_{1}(\theta)+F_{2}(\theta)$, with

$$
F_{1}(\theta)=F(\rho) \exp \left(-\frac{1}{2} b(\rho) \theta^{2}\right)\left(\frac{-i \Lambda \theta^{3}}{\log ^{2} \rho\left(\log ^{2} \rho+\theta^{2}\right)}+\cdots\right),
$$

an odd function of $\theta$ and

$$
F_{2}(\theta)=F(\rho) \exp \left(-\frac{1}{2} b(\rho) \theta^{2}\right)\left\{\frac{-\Lambda \theta^{4}}{\log ^{5} \rho}-\frac{\Lambda^{2} \theta^{6}}{2 \log ^{8} \rho}+O\left(\log ^{2} \rho+\frac{\theta^{12}}{\log ^{16} \rho}\right)\right\} .
$$

As $F_{1}(\theta)$ is an odd function, $\int_{-\delta}^{+\delta} D(\rho, \theta) d \theta=\int_{-\delta}^{+\delta} F_{2}(\theta) d \theta$. The integral can be computed, using repeated integrations by parts and one obtains $\int_{-\delta}^{+\delta} D(\rho, \theta) d \theta$ $=(2 \pi / b(\rho))^{1 / 2} F(\rho)\left\{-(3 / 16 \Lambda)(-\log \rho)+O\left(\log ^{2} \rho\right)\right\}$. Hence, by (15), assumption (b) of the lemma holds for $F(x)$ with $u(\rho)=-(3 / 16 \Lambda)(-\log \rho)$ and $\alpha=1$. Concerning assumption (a), one has from (7) that, for $\theta=\delta(\rho)$, $\left|F\left(\rho e^{i \delta}\right)\right|=F(\rho) \exp \left\{\Lambda \delta^{2} / \log \rho\left(\log ^{2} \rho+\delta^{2}\right)+O\left(\log ^{2} \rho\right)\right\}$ and, using (15) and the definition of $\delta(r)$,

$$
\begin{aligned}
\left|F\left(\rho e^{i \delta}\right)\right| & =F(\rho) \exp \left\{-\frac{1}{2} \delta(\rho)^{2} b(\rho)\left(1+\delta^{2} / \log ^{2} \rho\right)^{-1}+O\left(\log ^{2} \rho\right)\right\} \\
& =F(\rho) \exp \left\{-\Lambda \psi^{2}\left(1-\psi^{2} \log \rho+\psi^{4} \log ^{2} \rho\right)+O\left(\log ^{2} \rho\right)\right\} \\
& <F(\rho) \exp \left(-\frac{9}{10} \Lambda \psi^{2}\right)=F(\rho)(-\log \rho)^{18 / 5} \\
& =F(\rho) b(\rho)^{-1 / 2}(2 \Lambda)^{1 / 2}(-\log \rho)^{21 / 20}(1+\sigma(1))
\end{aligned}
$$


and, by (15),

$$
\left|F\left(\rho e^{i \delta}\right)\right|<(2 \Lambda / b(\rho))^{1 / 2} F(\rho)(\Lambda / n)^{21 / 20} .
$$

We now consider the circle of radius $r=\rho$, divided by a Farey dissection of order $N=\left[n^{3 / 8}\right]+1$. If $|\theta| \geqq \delta(\rho)$, from $\delta(\rho)=2^{1 / 2} \Lambda^{1 / 4} n^{-3 / 4}(\log n)^{1 / 2}(1+o(1))$ and $1 /(N+1)=n^{-3 / 8}(1+o(1))$ follows that $\theta$ may belong to any one of the three intervals $(\delta(\rho), 2 \pi /(N+1)),(2 \pi /(N+1), 2 \pi N /(N+1)),(2 \pi N /(N+1)$, $2 \pi-\delta(\rho))$. The first and last interval belong to the Farey arc centered at $x=\rho$. In that case, by (7),

$$
\begin{aligned}
F\left(\rho e^{i \theta}\right)=F(\rho) \exp \left\{\frac{\Lambda \theta^{2}}{\log \rho\left(\log ^{2} \rho+\theta^{2}\right)}+d \log (1\right. & \left.+\frac{i \theta}{\log \rho}\right) \\
& \left.+O\left(\log ^{2} \rho+\theta \operatorname{og} \rho+\theta^{2}\right)\right\} .
\end{aligned}
$$

Using (16), it follows for $|\theta|>\delta(\rho)$, that

$$
\begin{aligned}
\left|F\left(\rho e^{i \theta}\right) / F\left(\rho e^{i \delta}\right)\right|=\exp \left\{\frac{\Lambda}{\log \rho} \frac{\log ^{2} \rho\left(\theta^{2}-\delta^{2}\right)}{\left(\log ^{2} \rho+\theta^{2}\right)\left(\log ^{2} \rho+\delta^{2}\right)}\right. \\
\left.+d \log \left|1+\frac{i \theta}{\log \rho}\right|+O\left(\log ^{2} \rho+\theta^{2}\right)\right\} .
\end{aligned}
$$

We distinguish between the two cases:

$$
\begin{aligned}
& \delta<|\theta|<-\log \rho, \text { and (ii) }-\log \rho<|\theta|<2 \pi /(N+1) . \\
& \left|F\left(\rho e^{i \theta}\right) / F\left(\rho e^{i \delta}\right)\right|=\exp \left\{\frac{-\Lambda(-\log \rho)\left(\theta^{2}-\delta^{2}\right)}{\left(\log ^{2} \rho+\theta^{2}\right)\left(\log ^{2} \rho+\delta^{2}\right)}+O(1)\right\}
\end{aligned}
$$

hence, $\left|F\left(\rho e^{i \theta}\right) / F\left(\rho e^{i \delta}\right)\right|=O(1)$, because of $|\theta|>\delta(\rho)$.

$$
|\theta|>-\log \rho \text { implies } \theta^{2}-\delta^{2}>\log ^{2} \rho+\log ^{3} \rho .
$$

$\psi^{2}(\rho)=\log ^{2} \rho(1+o(1))$ and

$$
\begin{aligned}
\left|\frac{F\left(\rho e^{i \theta}\right)}{F\left(\rho e^{i \delta}\right)}\right| & <\exp \left\{\frac{\Lambda \log ^{3} \rho}{\left(\log ^{2} \rho+\theta^{2}\right)\left(\log ^{2} \rho+\delta^{2}\right)}-\frac{d}{4} \log (-\log \rho)+O(1)\right\} \\
& <\exp \left\{\frac{\Lambda \log \rho}{2 \theta^{2}}\left(1-\frac{\delta^{2}}{\log ^{2} \rho}\right)-\frac{d}{4} \log (-\log \rho)+O(1)\right\} \\
& <\exp \left\{\frac{-\Lambda}{2} \frac{(-\log \rho)}{4 \pi^{2}} n^{3 / 4}(1+o(1))\right\} \\
& =\exp \left\{\frac{-\Lambda^{3 / 2}}{8 \pi^{2}} n^{1 / 4}(1+o(1))\right\} \rightarrow 0 .
\end{aligned}
$$

Hence, if $\theta$ belongs to the first, or third interval, $F\left(\rho e^{i \theta}\right)=O\left(n^{-21 / 20} F(\rho) b(\rho)^{-1 / 2}\right)$ 
follows from (16). If $\theta$ belongs to the second interval, it is on a Farey arc corresponding to $h / k, 1<k \leqq N,(h, k)=1,1 / N \leqq h / k \leqq(N-1) / N$ and $\phi=\theta / 2 \pi-h / k$ satisfies (see 2.7 in [10]) $|k \phi|<1 / N$. By the remarks following (11), $\left|F\left(\rho e^{i \theta}\right)\right|<\exp \left\{-\Lambda t(\log \rho)^{-1}(1+o(1))+2 \Phi-2 C \pi k \phi+O(\log \rho)\right\}$. Using (13), $|k \phi|<1 / N$ and (15), $|2 \Phi-2 C \pi k \phi|=O\left(k \log k+k^{2} / N\right)=O(k \log k)$ $=O\left(n^{3 / 8+\eta}\right)=O\left\{(-\log \rho)^{-3 / 4-2 \eta}\right)$ for any $\eta>0$. It now follows that for $\theta$ on the second interval, $\left|F\left(\rho e^{i \theta}\right)\right| \leqq \exp \left\{\Lambda t(-\log \rho)^{-1}(1+o(1))\right\}$ $=\exp \left\{t(\Lambda n)^{1 / 2}(1+o(1))\right\}$, while, by (7) and (15), $n^{-1} b(\rho)^{-1 / 2} F(\rho)$ $=n^{-1} \exp \left\{(\Lambda n)^{1 / 2}(1+o(1))\right\}(2 \Lambda)^{-1 / 2}(\Lambda / n)^{3 / 4}$. Consequently, for any $|\theta|$ $\geqq \delta(\rho),\left|F\left(\rho e^{i \theta}\right)\right|=O\left(n^{-1} b(\rho)^{-1 / 2} F(\rho)\right)$, whence it follows that condition $(a)$ of the lemma is satisfied by $F(x)$ with $\alpha=1$. We now apply (14) to $F(x)$ $=\sum_{n=0}^{\infty} p_{n}(q) x^{n}$ and obtain, as $n \rightarrow \infty$, that $p_{n}(q)=\rho^{-n}(2 \pi b(\rho))^{-1 / 2} F(\rho)$ $\cdot\left(1-(3 / 16 \Lambda)(-\log \rho)+O\left(\log ^{2} \rho\right)\right)$. Replacing $F(\rho)$ and $b(\rho)$ by their values and using (15), one obtains

$$
\begin{array}{r}
p_{n}(q)=(2 \pi)^{-1 / 2(m+1)}(2 \Lambda)^{-1 / 2} q^{d} \cdot \prod_{j=1}^{m} \Gamma\left(\frac{a_{j}}{q}\right) \cdot\left(\frac{\Lambda}{n}\right)^{(d / 2+3 / 4)} e^{2(\Lambda n)^{1 / 2}} \\
\cdot\left(1-\left(\left(\frac{d+1}{2}\right)^{2}+K \Lambda-\frac{1}{16}\right)(\Lambda n)^{-1 / 2}+O\left(n^{-1}\right)\right) .
\end{array}
$$

As in $\S 3$, let $\lambda_{n}=(n-K)^{1 / 2}, E(x)=(2 \pi)^{-1 / 2} \Gamma(x), \omega=\prod_{j=1}^{m} E\left(a_{j} / q\right)$ and set $\lambda=\Lambda^{1 / 2}$. Then, if $I_{1}(x)$ stands for the Bessel function, (17) can also be written as

$$
p_{n}(q)=\frac{\omega}{q}\left(q \lambda / \lambda_{n}\right)^{d+1} \exp \left\{-\frac{d(d+2)}{4 \lambda \lambda_{n}}\right\} I_{1}\left(2 \lambda \lambda_{n}\right)\left(1+O\left(n^{-1}\right)\right)
$$

and this proves the first assertion of Theorem 2 .

9. For $H(x)$ one proceeds in essentially the same way. With $\mu=l \Lambda /(l+1)$, by (8) and (6), $a(r)=\mu(\log r)^{-2}-K l+O(\log r)$. The solution $\rho=\rho_{n}$ of $a(\rho)=n$ satisfies here

$$
\begin{aligned}
(\log \rho)^{-1} & =-\mu^{-1 / 2} n^{1 / 2}\left(1+\frac{1}{2} K l n^{-1}+O\left(n^{-3 / 2}\right)\right) \\
\log \rho & =-\mu^{1 / 2} n^{-1 / 2}\left(1-\frac{1}{2} K l n^{-1}+O\left(n^{-3 / 2}\right)\right) .
\end{aligned}
$$

Finally, $b(r)=2 \mu(-\log r)^{-3}+O(1)$. Setting $\delta=\delta(r)=(-\log r)^{3 / 2} \chi(r)$ with $\chi(r)=(-(4 / \mu) \log (-\log r))^{1 / 2}$, one verifies that $H(x)$ satisfies the assumptions (b) and (c) of the lemma, with $\alpha=1$ and $u(\rho)=-3(-\log \rho) / 16 \mu$. From (12) and (13) also follows that assumption (a) is satisfied with $\alpha=1$. We may now apply (14) to $H(x)=\sum_{n=0}^{\infty} p_{n}(q, l) x^{n}$ and obtain that 


$$
p_{n}(q, l)=\rho^{-n}(2 \pi b(\rho))^{-1 / 2} H(\rho)\left(1-\frac{3}{16 \mu}(-\log \rho)+O\left(\log ^{2} \rho\right)\right),
$$

or, replacing $H(\rho)$ and $b(\rho)$ by their values and using $\left(15^{\prime}\right)$,

$$
\begin{aligned}
p_{n}(q, l)= & \frac{1}{2} \pi^{-1 / 2}(l+1)^{-d} \mu^{1 / 4} n^{-3 / 4} \\
& \quad \cdot \exp \left(2(\mu n)^{1 / 2}\right)\left(1+\left(K l \mu-\frac{3}{16}\right) \mu^{-1 / 2} n^{-1 / 2}+O\left(n^{-1}\right)\right) .
\end{aligned}
$$

Setting $\nu_{n}=(n+K l)^{1 / 2}$ and $\nu=\mu^{1 / 2},(18)$ can also be written as

$$
p_{n}(q, l)=(l+1)^{-d} \frac{\nu}{\nu_{n}} I_{1}\left(2 \nu \nu_{n}\right)\left(1+O\left(n^{-1}\right)\right) .
$$

This finishes the proof of Theorem 2 .

10. A few particular cases are of interest.

(a) If $\{a\}=\{1,2, \cdots, q\}$ is the complete set of least positive residues $(\bmod q)$ one obtains the unrestricted partitions $p_{n}$. In this case $m=q, K$ $=1 / 24, d=1 / 2, \omega=\left\{2 \pi \prod_{a=1}^{q-1} 2 \sin (\pi a / q)\right\}^{-1 / 2}=(2 \pi q)^{-1 / 2}, \lambda_{n}=(n-1 / 24)^{1 / 2}$, $\lambda=\pi / 6^{1 / 2}$ and $\left(17^{\prime}\right)$ becomes

$$
\begin{aligned}
p_{n}(q)= & p_{n}=\left(2 \pi^{-1 / 2}\right)\left(6 \lambda_{n}^{2}\right)^{-3 / 4} \\
& \cdot \exp \left(\frac{-5\left(6^{1 / 2}\right)}{16 \pi \lambda_{n}}\right) I_{1}\left(\pi(2 / 3)^{1 / 2} \lambda_{n}\right)\left(1+O\left(n^{-1}\right)\right) .
\end{aligned}
$$

This is indeed in agreement with the result of Rademacher ((7.3)) in [10] and both expressions can be written in the form

$$
p_{n}=\frac{1}{4(3)^{1 / 2} n} e^{\pi(2 / 3)^{1 / 2}(n)^{1 / 2}}\left(1-\left(\frac{6(2)^{1 / 2}}{\pi}+\frac{\pi}{6 \cdot 2^{1 / 2}}\right) \frac{3^{1 / 2}}{12} n^{-1 / 2}+O\left(n^{-1}\right)\right) .
$$

However, if Rademacher's formula is written in the form of $\left(17^{\prime \prime}\right)$, the error term is $O\left(e^{-\sigma(n)^{1 / 2}}\right)$ with constant $\sigma>0$.

(b) If $d=0,\left(17^{\prime}\right)$ becomes

$$
p_{n}(q)=\frac{\omega \lambda}{\lambda_{n}} I_{1}\left(2 \lambda \lambda_{n}\right)\left(1+O\left(n^{-1}\right)\right) .
$$

In particular, $d=0$, holds if $\{a\}$ is symmetrical; in this case, $\left(17^{\prime \prime \prime}\right)$ is easily identified with Petersson's formula (10.8) in [8], except for the order of its error term.

(c) Setting $d=0$ in $\left(18^{\prime}\right)$ one obtains $p_{n}(q, l)=\left(\nu / \nu_{n}\right) I_{1}\left(2 \nu \nu_{n}\right)\left(1+O\left(n^{-1}\right)\right)$, again a particular case of (10.8) in [8].

In general, one observes that the error terms $O\left(n^{-1}\right)$ in $\left(17^{\prime}\right)$ and $\left(18^{\prime}\right)$ are 
actually of order $O\left(e^{-\sigma(n)^{1 / 2}}\right), \sigma>0$, as well in the case of symmetric sets $\{a\}$, when $d=0$, as also in case $\{a\}=\{1,2, \cdots, q\}$, when $d=1 / 2$. Hence, one may state as a plausible

CONJECTURE. The error terms in $\left(17^{\prime}\right)$ and $\left(18^{\prime}\right)$ are actually of order $O\left(e^{-\sigma(n)^{1 / 2}}\right)$, with $\sigma>0$.

11. Proof of Corollary 1 . In case $q \equiv 1(\bmod 4)$ the sets of quadratic residues and of quadratic nonresidues are both symmetrical. Furthermore, $m=(q-1) / 2, d=0, \Lambda=\pi^{2}(q-1) / 12 q$,

$$
K=K^{+}=(1 / 24 q) \sum_{(a / q)=+1}\left(q^{2}-6 a q+6 a^{2}\right)=-q(q-1) / 24+(1 / 4 q) \sum_{(a / q)=+1} a^{2}
$$

in the case of quadratic residues and, similarly, $K=K^{-}=-q(q-1) / 24$ $+(1 / 4 q) \sum_{(a / q)=-1} a^{2}$ in the case of nonresidues. From $\left(17^{\prime \prime}\right)$ now follows:

$$
\begin{aligned}
p_{n}^{ \pm}(q)= & 2^{-(q+1) / 4}\left(\frac{q-1}{3 q}\right)^{1 / 4}\left\{\prod_{(a / q)= \pm 1} \sin \frac{a}{q}\right\}^{-1 / 2} n^{-3 / 4} \\
& \cdot \exp \left\{\frac{\pi}{3}\left(\frac{3(q-1)}{q}\right)^{1 / 2} n^{1 / 2}\right\} \\
& \cdot\left(1-\frac{\pi}{6}\left(\frac{3(q-1)}{q}\right)^{1 / 2}\left(K^{ \pm}-\frac{9 q}{4 \pi^{2}(q-1)}\right) n^{-1 / 2}+O\left(n^{-1}\right)\right),
\end{aligned}
$$

where either + or - has to be taken throughout both members. Taking the ratio, one observes (see [4]) that $\left\{\prod_{a \bmod q}(\sin a / q)^{(a / q)}\right\}^{-1 / 2}=\epsilon^{h}$, so that

$$
\frac{p_{n}^{+}(q)}{p_{n}^{-}(q)}=\epsilon^{h}\left(1-\frac{\pi}{6}\left(\frac{3(q-1)}{q}\right)^{1 / 2}\left(K^{+}-K^{-}\right) n^{-1 / 2}+O\left(n^{-1}\right)\right) .
$$

However, $K^{+}-K^{-}=(1 / 4 q) \sum_{a \bmod q}(a / q) a^{2}$. This sum is well known (see [11, vol. 2, pp. 677-678] and [1]) and equals $4 c q \alpha_{5}(q)$. This finishes the proof of (1). Similarly, from (18), with the same values of the constants, for $n \rightarrow \infty$,

$$
\begin{aligned}
& p_{n}^{ \pm}(q, l)=\left(\frac{l}{l+1} \frac{q-1}{12 q}\right)^{1 / 4} n^{-3 / 4} \exp \left\{\frac{\pi}{3}\left(\frac{l}{l+1} \frac{3(q-1)}{q}\right)^{1 / 2} n^{1 / 2}\right\} \\
& \left(1+\frac{\pi}{6}\left(\frac{3(q-1)}{q} \frac{l}{l+1}\right)^{1 / 2}\left(K^{ \pm \cdot l}-\frac{9}{4 \pi^{2}} \frac{l+1}{l} \frac{q}{q-1}\right) n^{-1 / 2}+O\left(n^{-1}\right)\right) .
\end{aligned}
$$

Taking ratios,

$$
\frac{p_{n}^{+}(q, l)}{p_{n}^{-}(q, l)}=1+\frac{\pi}{6}\left(\frac{3(q-1)}{q} \frac{l}{l+1}\right)^{1 / 2} \cdot l\left(K^{+}-K^{-}\right) n^{-1 / 2}+O\left(n^{-1}\right)
$$

and, replacing $K^{+}-K^{-}$by its value $c \alpha_{5}(q)$, one obtains (2), thus finishing the proof of Corollary 1. 
12. Proof of Corollary 2. If $q \equiv 3(\bmod 4), q>3$ and $\{a\}$ stands for the set of quadratic residues, or nonresidues, respectively, $m=(q-1) / 2$ and $d^{ \pm}=1 / q \sum_{(a / q)= \pm 1} a-(q-1) / 4$, where the signs + or - have to be taken the same in both members and refer to quadratic residues, or nonresidues, respectively. (17) yields

$$
\begin{aligned}
& \frac{p_{n}^{+}(q)}{p_{n}^{-}(q)}=\left(\frac{q^{2} \Lambda}{n}\right)^{\left(d^{+}-d^{-}\right) / 2} \prod_{a \bmod q}\left\{\Gamma\left(\frac{a}{q}\right)\right\}^{(a / q)} \\
& \quad\left(1-\left(\left(\frac{d^{+}+1}{2}\right)^{2}-\left(\frac{d^{-}+1}{2}\right)^{2}+\Lambda\left(K^{+}-K^{-}\right)\right)(\Lambda n)^{-1 / 2}+O\left(n^{-1}\right)\right) .
\end{aligned}
$$

One has now (see [4]) for $q>3$, that $d^{+}-d^{-}=(1 / q) \sum_{a \bmod q}(a / q) a=-h$ $=-2 h / w$ where $h$ is the class number of the quadratic field $R\left((-q)^{1 / 2}\right)$, and $w=2$ is the number of its roots of unity. Also,

and

$$
K^{ \pm}=(1 / 24 q) \sum_{(a / q)= \pm 1}\left(q^{2}-6 a q+6 a^{2}\right)=q(q-1) / 48
$$

$$
\begin{aligned}
\frac{p_{n}^{+}(q)}{p_{n}^{-}(q)}= & \prod_{a \bmod q}\left\{\Gamma\left(\frac{a}{q}\right)\right\}^{(a / q)} \\
& \quad\left(\frac{12 n}{\pi^{2} q(q-1)}\right)^{h / w}\left(1-\frac{1}{\pi}\left(\frac{6 q}{(q-1)}\right)^{1 / 2} \frac{h n^{-1 / 2}}{w}+O\left(n^{-1}\right)\right) .
\end{aligned}
$$

In exactly the same way, using (18) instead of (17), one obtains

$$
\frac{p_{n}^{+}(q, l)}{p_{n}^{-}(q, l)}=(l+1)^{-d^{+}+d^{-}}\left(1+O\left(n^{-1}\right)\right)=(l+1)^{h}\left(1+O\left(n^{-1}\right)\right) .
$$

In case $q=3,(7)$ and (8) still hold, although the proof has to be changed in some details; also the lemma remains applicable. Hence, one obtains as before (17) and (18). Now, however, $d^{+}=1 / 3-1 / 2=-1 / 6, d^{-}=2 / 3-1 / 2=1 / 6$, so that $d^{+}-d^{-}=-1 / 3$. As the number $w$ of roots of unity in $R\left((-3)^{1 / 2}\right)$ is $w=6$ and $h=1$, this finishes the proof of Corollary 2.

\section{BIBLIOGRAPHY}

1. G. H. Hardy, On the representation of a number as a sum of any number squares, Trans. Amer. Math. Soc. vol. 21 (1920) pp. 255-284.

2. G. H. Hardy and S. Ramanujan, Asymptotic formulae in combinatorial analysis, Proc. London Math. Soc. (2) vol. 17 (1918) pp. 75-115.

3. W. K. Hayman, A generalization of Stirling's formula, J. Reine Angew. Math. vol. 196 (1956) pp. 67-95.

4. E. Hecke, Vorlesungen uber die Theorie der algebr. Zahlen, Leipzig, Akademische Verlagsgeselschaft, 1923.

5. J. Lehner, Partition function connected with the modulus five, Duke Math. J. vol. 8 (1941) pp. 631-655. 
6. J. Livingood, $A$ partition function with the prime modulus $p>3$, Amer. J. Math. vol. 67 (1945) pp. 194-208.

7. G. Meinardus, Asymptotishe Aussagen ïber Partitionen, Math. Z. vol. 59 (1954) pp. 388-398.

8. H. Petersson, Ueber Modulfunktionen u. Partitionenprobleme, Abh. Deutsch. Akad. Wiss. Berlin Kl. Math. Nat. (1954) no. 2.

9. - Ueber die arithmetischen Eigenschaften eines Systemes multiplikativer Modulfunktionen von Primzahlstufe, Acta Math. vol. 95 (1956) pp. 57-110.

10. H. Rademacher, On the Partitionfunction $p(n)$, Proc. London Math. Soc. (2) vol. 43 (1937) pp. 241-254.

11. - The Fourier coefficients of the modular invariant $J(\tau)$, Amer. J. Math. vol. 60 (1938) pp. 501-512.

12. H. J. Smith, Collected Math. Papers, Clarendon Press, Oxford, 1894.

University of Pennsylvania, Philadelphia, Pa. 